\title{
Renta petrolera y dependencia económica. El papel de China en los nuevos procesos de crecimiento en África: el caso sudanés (1989-2011)
}

\author{
Oil revenues and economic \\ dependency. China's role in \\ Africa's new growth processes: \\ A case study of Sudan (1989-2011)
}

\section{ALFREDO LANGA HERRERO* DANIEL COQ HUELVA**}

Resumen: Este artículo analiza el crecimiento de la economía sudanesa de 1989 a 2011 desde la confluencia de cuatro procesos: los efectos del neoliberalismo en África y el mundo árabe, el proyecto político islamista, la extracción masiva de petróleo y los cambios en el marco geoestratégico global marcado por el creciente papel de China como inversor y cliente de las economías africanas. En este desarrollo se distinguen dos fases: en la primera se sientan las bases de la explotación y la distribución de crudo y se llevan a cabo importantes inversiones en infraestructura; en la segunda se asiste a la consolidación de Sudán como país petrolero, lo que conlleva la transformación de la estructura productiva y del papel del Estado sudanés. El texto estudia, además, el impacto del petróleo, del cambiante contexto geopolítico global

Recepción: 18 de mayo de 2018. / Aceptación: 15 de noviembre de 2018.

* Universidad Alice Salomon Berlin, alfredo.langa@iecah.org

** Universidad de Sevilla, dcoq@us.es 
y, particularmente, del creciente papel de China en la evolución del escenario de conflicto armado.

Palabras clave: petróleo; dependencia; conflicto armado; China; islamismo; Sudán.

Abstract: This article analyzes the growth of the Sudanese economy from 1989 to 2011, as a result of four concurrent processes: neoliberalism in Africa and the Arab world, Islamism, extractivism, and changes in the global geopolitical framework strongly influenced by China's growing role. Two phases have been identified in this process: in the first phase, the foundations were laid for the exploitation and distribution of crude oil and large investments are made in infrastructure. In the second, Sudan consolidated its role as an oil-producing country, entailing major transformations in its productive structure and in the State's role. The authors also examine the impact of oil, the shifting global geopolitical context, particularly in regard to China in the evolution of the armed conflict scenario.

Keywords: oil; dependency; armed conflict; China; Islamism; Sudan.

\section{Introducción}

Sudán es un lugar de confluencia entre el mundo árabe y África subsahariana que, en los últimos años, ha sufrido convulsos procesos sociopolíticos marcados por una fuerte violencia (Jok, 2001; Beswick, 2004; Elnur, 2009). Como resultado, sus bases socioeconómicas se han visto profundamente afectadas. En este contexto, el objetivo de este trabajo es analizar la especialización productiva y la acumulación experimentadas por la economía sudanesa poniéndolas en relación, por un lado, con las transformaciones sociopolíticas internas y, por otro, con las modificaciones de la posición sudanesa ${ }^{1}$ en el conjunto de las relaciones geopolíticas internacionales.

Con esta finalidad, se hace una aproximación de corte realista-crítico basada en la observación y la sistematización de

\footnotetext{
${ }^{1}$ Este texto se refiere a la República de Sudán existente hasta julio de 2011, fecha en que se independizaron los territorios del sur y se fundó la República de Sudán del Sur.
} 
los elementos sociales, políticos y geoestratégicos que influyen estrictamente en la evolución económica de Sudán (Lawson, 1997 y 2003; Sayer, 1995; Bashkar, 2011). Por lo tanto, aunque centrado en lo económico, el análisis tiene un modesto pero perceptible componente multidisciplinar y coevolutivo (Nelson, 2006; Gual y Norgaard, 2010). Como consecuencia, en la explicación emergen conceptos (neoliberalismo, islamismo, extractivismo, etcétera) esenciales para interpretar los cambios socioeconómicos en Sudán. Hay que resaltar que se llegó a éstos de forma inductiva y no constituyen un a priori de la investigación, sino su producto. En este sentido, una de las principales aportaciones del artículo es propiciar la confluencia de líneas de razonamiento teórico relativamente independientes entre sí, como las reflexiones sobre neoliberalismo (Larner, 2003; Harvey, 2007; Brenner, Peck y Theodore, 2010), islamismo (Bayat, 2005) o extractivismo (Gudynas, 2009; Svampa, 2011). Es también esta aproximación realista y coevolutiva la que justifica la incorporación de un enfoque desde las relaciones internacionales. En el caso sudanés, no se entenderían las transformaciones de su base económica si no se toma en cuenta el cambiante contexto internacional, concretamente el creciente papel de China en el conjunto del continente africano.

Otro elemento fundamental en cualquier acercamiento realista es el periodo de análisis y su división en etapas. En este caso, el marco cronológico corresponde a 1989-2011. Como se demostrará, aparte de la limitación de espacio inherente a cualquier artículo científico, varias razones justifican esta decisión. En 1989 llegó al poder el Frente Nacional Islámico, partido que impuso fuertemente la islamización al tiempo que liberalizó y privatizó distintos sectores económicos. Se cierra en 2011 el marco temporal, ya que la independencia de Sudán del Sur supuso un cambio esencial desde el punto de vista político y económico, con el nacimiento de un nuevo Estado.

Puede afirmarse, por lo tanto, que el periodo 1989-2011 resume el largo ciclo de hegemonía islamista en Sudán, con los cambios sociales, económicos y geoestratégicos que lleva aparejado. El ciclo abarca dos fases definidas: de 1989 a 1999, caracterizada por las reformas económicas estructurales y la profundización del islamismo político, y de 1999 a 2011, en que 
comienza el “divorcio” entre el elemento más político y el más militar del islamismo, al tiempo que se produce un fuerte auge petrolero.

Las bases empresariales y geopolíticas que permiten el desarrollo petrolero se analizan en el segundo punto. Entre los distintos elementos geopolíticos que confluyen en el rápido crecimiento de la producción sudanesa del hidrocarburo está el papel que desempeña China. En el tercer apartado se investiga cómo el nuevo modelo de producción no sólo modifica la estructura de las relaciones exteriores y el conjunto de la economía, sino que también se relaciona con la nueva posición en los circuitos económicos globales. En el punto cuarto se estudian el papel del Estado sudanés como consecuencia de los cambios políticos internos y el desarrollo de la especialización petrolera. En el quinto apartado se revisa la forma en que la dinámica del conflicto armado interno ha condicionado históricamente la expansión de la extracción petrolera y cómo los fondos procedentes de este negocio han afectado la evolución de dicho conflicto. Finalmente, el sexto apartado corresponde a las conclusiones sobre los procesos socioeconómicos vividos por Sudán en los últimos años, con base en fuentes de información secundarias y estadísticas de fuentes contrastadas, aunque para el caso sudanés hay que tener en cuenta la dificultad de acceso a datos oficiales.

\section{El desarrollo de la especialización petrolera a partir de los años noventa: elementos políticos internos y externos}

Desde mediados de la década de 1970 se sabe que en Sudán hay importantes reservas de petróleo, pero, como se observa en la gráfica 1 , no fue sino hasta finales de los años noventa cuando el país se convirtió en productor relevante. Sin embargo, el inicio de la actividad precisa del desarrollo previo de una importante actividad inversora en tareas de exploración, construcción de infraestructura, puesta en explotación de los pozos y creación de un sistema de distribución, acciones que se desarrollaron a lo largo de los años noventa.

En el caso de Sudán, dichas labores se encontraban sujetas a riesgos importantes por el escenario de violencia que predo- 
GRÁFICA 1. Producción de crudo de 1997 a 2011 en miles de barriles diarios

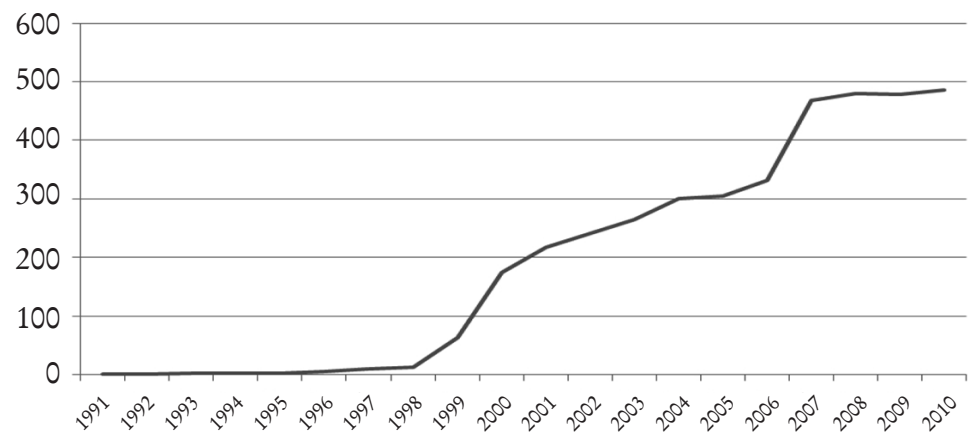

Fuente: EIA, 2013, y elaboración propia.

minaba en muchas de las zonas productoras (Le Billon, 2001; Rone, 2003; Patey, 2007).

El periodo 1989-1999: el asentamiento de las bases de la explotación petrolera

En 1989, una coalición cívico-militar islamista liderada por el Frente Nacional Islámico, con Hassan al Turabi como principal dirigente e ideólogo, conquistó el poder con un discurso centrado en la regeneración política y espiritual del país, asociado, a su vez, a una transformación radical de sus estructuras económicas, sociales y políticas. En este contexto, los grupos dirigentes tradicionales - musulmanes vinculados a las cofradías sufíes - fueron desplazados del poder (El-Affendi, 1991; Burr y Collins, 2003).

Como consecuencia, se produjeron despidos masivos en la administración pública y en el ejército (Musa, 2001). Simultáneamente, como se verá más adelante, tanto la recaudación fiscal como el gasto público se contrajeron fuertemente, ya que el ideal islamista se basa en enérgicas reducciones impositivas debido a que vinculan una presión fiscal elevada con formas "occidentales" de socialismo (Warburg, 2003). En este pun- 
to se observa una primera conexión entre el neoliberalismo propugnado por las instituciones financieras internacionales (Fondo Monetario Internacional y Banco Mundial) y el proyecto político islamista impulsado por el Frente Nacional Islámico.

No obstante, dicho proyecto también precisaba, en algunos puntos, un Estado fuerte, ya que uno de sus conceptos clave era el tamakeen o empoderamiento, entendido como la consolidación gradual de un poder que se legitima sobre bases religiosas y que se asocia al desarrollo de un Estado con proyección exterior y capacidad de ordenar interiormente las relaciones sociales (Elnur, 2009; Sharfi, 2015). En medio de la reducción de la carga fiscal, la única vía para propiciar el tamakeen era obtener financiación recurriendo al aumento de las ventas en los mercados exteriores. Éste fue el papel esencial del petróleo.

El acercamiento islamista al petróleo fue pragmático, apoyado en un concepto básico de su filosofía político-religiosa: la figh eldarura o "teología de la necesidad" (Warburg, 2003; Elnur, 2009), que permite realizar alianzas con aquellos grupos u organizaciones que se consideren necesarios para el fortalecimiento de la propia posición. La "necesidad" es múltiple: de fondos para sostener al Estado y dinamizar la economía, de capitales para afirmar el proyecto islamista frente a grupos e ideas rivales, y de hacer frente a agentes internacionales considerados hostiles. En este sentido, los islamistas tuvieron una mala relación con Estados Unidos casi desde su llegada al poder, que terminó concretándose en el embargo comercial y de inversiones a Sudán en 1997.

El pragmatismo petrolífero islamista derivado de la aplicación de la teología de la necesidad se tradujo, por una parte, en la apertura de la exploración y la explotación a empresas occidentales, pero procedentes de países relativamente periféricos, como Canadá o Suecia (World Bank, 2003; Kobryn, 2004). Por otra parte, también propició que compañías procedentes de Asia, y especialmente de China, participaran en el negocio. El principio de no injerencia en asuntos internos, defendido por la política exterior china, es totalmente coherente con el principio islamista del tamakeen (Sharfi, 2015), por lo que, desde el comienzo de los noventa, se produjo una convergencia entre 
los intereses del emergente capitalismo chino y los de los islamistas sudaneses.

En todo caso, la aproximación a China por parte de los islamistas no ha sido espontánea ni reactiva, sino más bien cautelosa y gradual, acompañada de fuertes debates en la coalición gubernamental sobre su conveniencia (Large, 2008). Por ello, se hizo todo lo posible por diversificar a los agentes participantes $\mathrm{y}$ las fuentes de financiación, en un intento de relacionarse no sólo con China, sino también con otros países del Asia Oriental, como Malasia, considerado estratégico por su doble condición de asiático-oriental y musulmán, una de cuyas empresas, Petronas Carigali, tiene gran protagonismo (cuadro 1).

En definitiva, en la década de 1990 Sudán aún no producía petróleo, pero se sentaron las bases institucionales y empresariales que harían posible el incremento de la extracción en el posterior decenio. Al respecto, en el cuadro 1 se

CuAdro 1. Concesiones petroleras en Sudán en 2002

\begin{tabular}{|c|c|c|c|}
\hline $\begin{array}{l}\text { Bloque } \\
\text { y yacimientos }\end{array}$ & $\begin{array}{c}\text { Empresa } \\
\text { concesionaria }\end{array}$ & País & $\begin{array}{c}\text { Participación } \\
\text { (\%) }\end{array}$ \\
\hline \multirow{4}{*}{$\begin{array}{l}1 \text { (Unity), } \\
2 \text { (Heglig) } \\
\text { y } 4 \text { (Kaikang) }\end{array}$} & Talisman & Canadá & 25 \\
\hline & Petronas Carigali & Malasia & 30 \\
\hline & Sudapet & Sudán & 5 \\
\hline & CNPC & China & 40 \\
\hline \multirow{5}{*}{$\begin{array}{l}3 \text { (Adar) y } 7 \\
\text { (Melut) }\end{array}$} & Gulf Petroleum Corporation & Qatar & 6 \\
\hline & Petronas Carigali & Malasia & 40 \\
\hline & CNPC & China & 41 \\
\hline & Sudapet & Sudán & 8 \\
\hline & Thani Corporation & Emiratos Árabes & 5 \\
\hline \multirow{4}{*}{$5 a$ y $5 b$} & Lundin Oil/IPC & Suecia & 24.5 \\
\hline & Petronas Carigali & Malasia & 41 \\
\hline & OMV Sudan Exploration & Austria & 24.5 \\
\hline & Sudapet & Sudán & 10 \\
\hline 5 o b (Central) & Total Fina Elf & Bélgica y Francia & 100 \\
\hline 6 & CNPC & China & 100 \\
\hline
\end{tabular}

Fuente: Human Rights Watch, 2003, y Middle East Economic Survey, 2003. 
MAPA 1. Recorrido del oleoducto sudanés en 2007

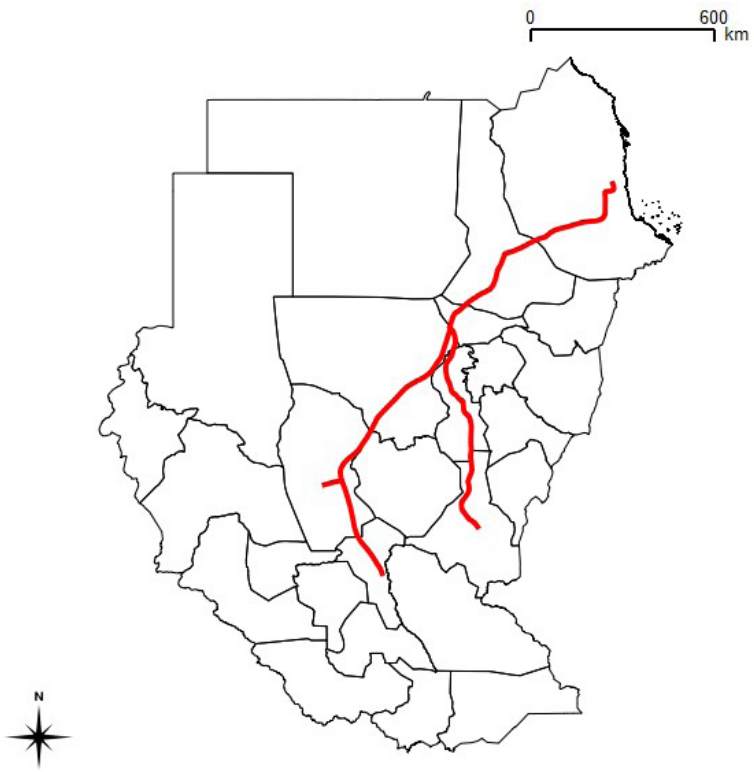

Fuente: EIA, 2007, y elaboración propia.

muestran los principales actores del todavía emergente sector petrolero.

Los años en los que se reconocieron las concesiones ponen de manifiesto la forma en que el gobierno islamista encontró nuevos socios internacionales y el creciente papel de China en este contexto. Las inversiones desarrollaron no sólo la extracción de petróleo, sino también el conjunto de su cadena de valor. Así, a partir de 1997, se acordó la construcción de un oleoducto (mapa 1) que llevaría el crudo hacia Port Sudán (Rone, 2003; World Bank, 2003). Esta movilización de inversión privada es especialmente valorable por dos razones: no es frecuente que se desarrolle toda la cadena de valor - muchos países tienen dificultades, por ejemplo, con el refinado de sus productos- y se requieren inversiones importantes en un país con graves problemas de liquidez. 
El periodo 2000-2011. La extracción masiva de petróleo del suelo sudanés

La característica fundamental de esta etapa es que el petróleo no es ya una promesa, sino una realidad. El primer año en que la extracción en Sudán tuvo un volumen relativamente importante fue 1999, cuando alcanzó los 69 millones de barriles. Un año más tarde se multiplicó casi por tres. Al final de la década llegó a 487 millones, lo que convirtió a Sudán en un productor de mediana relevancia mundial, aunque se encontraba todavía lejos de países como Nigeria, Angola, Argelia o Libia (World Bank, 2012).

El inicio de la actividad petrolera coincidió con un cambio de gobierno que prescindió de la rama política islamista, representada por el Frente Nacional Islámico y su líder Hassan al Turabi, con el fin de fortalecer el elemento militar, encarnado en el presidente Bashir (Burr y Collins, 2003). Esta "militarización" del régimen estaba vinculada, entre otras cosas, a las propias dinámicas abiertas por la producción petrolífera. El aumento de la extracción se asoció al número de áreas productoras, muchas situadas en el sur, lo que conllevó una profundización en el conflicto armado interno. El petróleo, al suponer un acceso directo a flujos financieros que podían ser utilizados en el afán bélico, se transformó en uno de los principales vectores explicativos de la guerra (Reeves, 2002; Patey, 2007). Esto se produjo en un momento en que Estados Unidos presionaba al gobierno sudanés debido a las denuncias internacionales de connivencia de Jartum con movimientos armados y terroristas del mundo árabe y musulmán (Large, 2008).

En todo caso, el gran crecimiento de las rentas obtenidas por el petróleo dio un mayor margen de maniobra al gobierno que se tradujo en un protagonismo ascendente de la empresa pública Sudapet. El enfrentamiento con Estados Unidos llevó a un fortalecimiento de la presencia de empresas chinas y otros inversores asiáticos en las nuevas concesiones. Sin embargo, esta notoriedad china implicó contradicciones respecto al principio de no intervención, ya que la cooperación chinosudanesa incluyó un componente militar concretado en la venta de armas y, sobre todo, en la transferencia de tecnología 
MAPA 2. Red ferroviaria de Sudán en 2004 y situación de la presa de Merowe

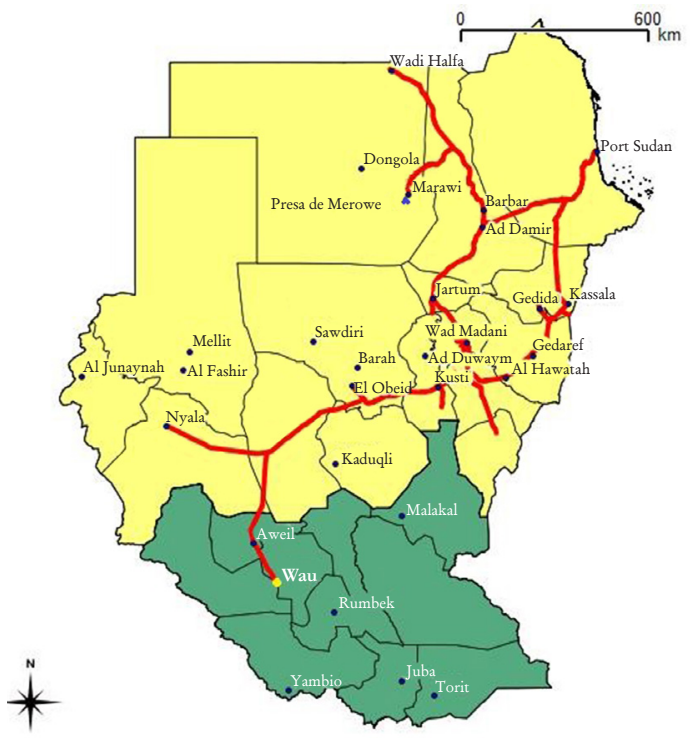

Fuente: elaboración propia.

destinada al complejo industrial-militar sudanés. Además, la creciente disponibilidad de divisas por parte del gobierno alteró, se quiera o no, el inestable equilibrio en la zona (Large, 2008). Esta capacidad china de influir se hizo especialmente evidente con la intensificación de la violencia en Darfur (Large, 2007; Parello-Plesner y Duchâtel, 2014).

China también adquirió un papel central como inversor y socio comercial de otras economías africanas especializadas en la producción de materias primas en general, y de petróleo en particular (Taylor, 2006). Por ello, el gobierno islamista, aun reconociendo la primacía de aquel país, promovió la apertura a agentes de otras naciones, en especial de India, más allá del papel tradicionalmente desempeñado por Malasia. Se continuó, además, con la idea de desarrollar el conjunto de la cadena de valor. La puesta en explotación de nuevos pozos se acompañó 
con otra infraestructura como, por ejemplo, la ampliación de la red ferroviaria (mapa 2), la construcción de la presa de Merowe o la extensión del oleoducto (mapa 1). Es decir, se avanzó en la vía abierta con la aplicación de los principios pragmáticos de la teología de la necesidad, tendentes a redefinir el marco de relaciones geopolíticas y económicas de Sudán, al vincular el país a la nueva área de expansión de Asia y al alejarlo del tradicional esquema de relaciones euronorteamericano.

\section{La transformación de las estructuras productivas y del comercio exterior}

La transformación de los agentes inversores encargados de desarrollar la especialización petrolera, como se ha visto, ha sido un elemento clave en el tamekeen. En esta línea, otro aspecto fundamental es la evolución de la estructura del comercio exterior. Por un lado, el mecanismo importador es capaz de condicionar el ritmo de muchas de las actividades económicas internas, pues se ven afectadas por restricciones a la renovación de la maquinaria, por ejemplo, y, por otro, las exportaciones determinan las fuentes de financiación externa de una economía con fuertes necesidades de ésta.

Importaciones, exportaciones y crecimiento durante la década de 1990

Hasta el comienzo de la explotación petrolífera en 1999, Sudán se caracterizaba por un relativamente débil sector exterior. Tenía escasa capacidad exportadora - alrededor de 5\% del PIB a principios de los noventa-, basada además en pocos productos agrícolas (algodón, sésamo) y ganado, tradicionalmente destinados a Europa y a países árabes de renta elevada, como Arabia Saudí. Pese a esta limitación, en términos reales el rendimiento obtenido por esta vía progresó a una media de $13 \%$ anual acumulativo entre 1989 y 1999 (World Bank, 2003, 2009 y 2012).

Sin embargo, de acuerdo con un patrón clásico en el funcionamiento de economías periféricas, esto no resultó suficiente 
para equilibrar el sector exterior. Las necesidades de importación eran también muy elevadas y crecieron a una media anual de $8.8 \%$ entre 1989 y 1999. Estas importaciones se concentraban en las manufacturas, cuya participación media en el total se situó en algo más de 63\% durante la década de 1990 (World Bank, 2003, 2009 y 2012). No se observaron procesos relevantes de sustitución de importaciones y, es más, las manufactureras se dispararon en relación con la producción interna. Esta situación generó constantes déficits exteriores y explica la sucesiva aplicación de programas de ajuste estructural negociados con el Fondo Monetario Internacional (World Bank, 2003; Berhanu, 2011).

Hay, empero, un sector donde sí se aplicaron políticas de sustitución de importaciones: la industria militar. La escasez crónica de divisas limitaba considerablemente las importaciones de armamento y, con ello, las posibilidades efectivas de control de las guerrillas del sur del país. La imposibilidad de acabar con la guerra civil y la falta de una dirección efectiva en amplias zonas del sur eran fuente continua de desgaste para el gobierno islamista, con un intenso componente nacionalista. Por ello, en 1993 se promovió la creación de la Military Industry Corporation, complejo que incluía fábricas de armas y municiones, así como aparatos electrónicos y de telecomunicaciones. Sudán contó con la asistencia tecnológica y el uso de patentes de China, Irán y Rusia (HSBA, 2014).

El acceso de los islamistas al poder en 1989 no supuso ningún cambio sustancial en los sectores importadores y exportadores ni con los países con los que se comerciaba. Por lo tanto, al contrario de lo que ocurría en la atracción de inversiones en el sector del petróleo, las relaciones comerciales y financieras de la economía sudanesa tuvieron en el periodo 1989-1999 un perfil esencialmente continuista, como muestra la gráfica 2 (Woodward, 1994).

\section{El periodo 1999-2011}

A partir de 1999, la estructura exportadora sudanesa se modificó radicalmente. Las exportaciones totales pasaron de 780 millones de dólares en 1999, a 11404 en 2010. Esta progresión 
GRÁficA 2. Tasa de apertura y saldo de la balanza comercial en relación con el PIB (\%)

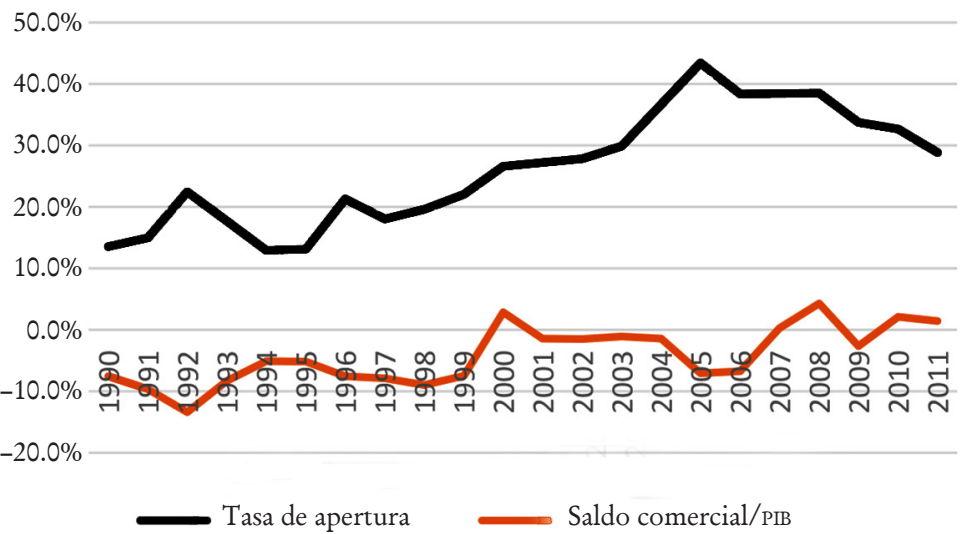

Fuente: elaboración propia a partir de datos del Banco Mundial (World Bank, 2003, 2009 y 2012).

se explica por las ventas de petróleo, que supusieron más de $90 \%$ del total de las exportaciones en 2010. De esta manera se transitó con rapidez a una situación de monoexportación y se asistió al desarrollo de una forma especialmente acusada de extractivismo. Sudán pasó de vender algodón a vender petróleo (gráfica 3).

La actividad petrolera contribuyó, además, a acentuar la dualidad de la estructura productiva sudanesa. De este modo, se distingue una parte "moderna”, muy capitalizada y dirigida al mercado exterior, del conjunto de tareas poco capitalizadas de subsistencia que suponen la base de la economía del país. Las industrias extractivas (entre las que se incluye la petrolera) suponían $22.5 \%$ del PIB en 2010. Frente a ello, se contraponen sectores marcadamente tradicionales, como la agricultura o parte importante de los servicios (gráfica 4).

Al ser China el comprador casi exclusivo del petróleo sudanés, se volvió en el gran financiador externo de la economía del país. Así, no sólo era el principal inversor, sino también el principal mercado del petróleo y, por lo tanto, de las exportaciones sudanesas (gráfica 5). 
GRÁFICA 3. Importaciones y exportaciones de Sudán, 1990-2011 (millones de dólares)

14000.0

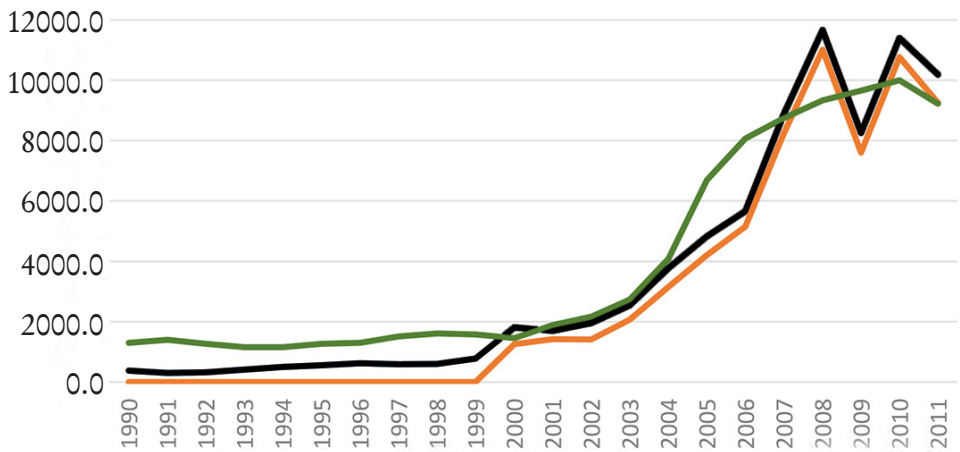

_Exportaciones totales Exportaciones de combustible Importaciones

Fuente: elaboración propia a partir de datos del Banco Mundial (World Bank, 2003, 2009 y 2012).

GrÁFICA 4. Evolución de la distribución de las exportaciones por producto en 1998, 2000, 2004, 2008 y 2011

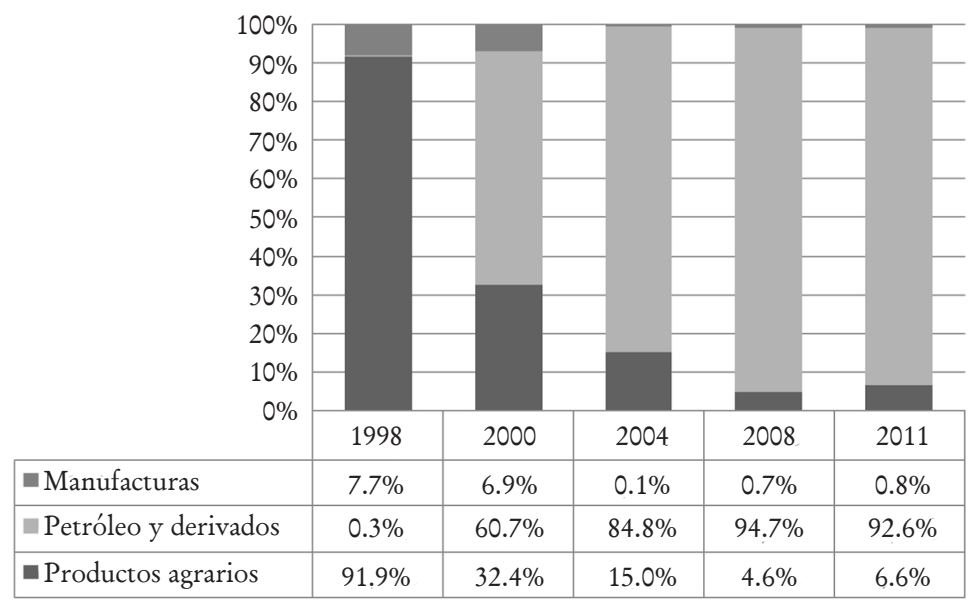
2003).

Fuente: elaboración propia a partir de datos del Banco Mundial (World Bank, 
GRÁFICA 5. Evolución de la distribución de las exportaciones, 1999, 2000, 2005 y 2010, según destino

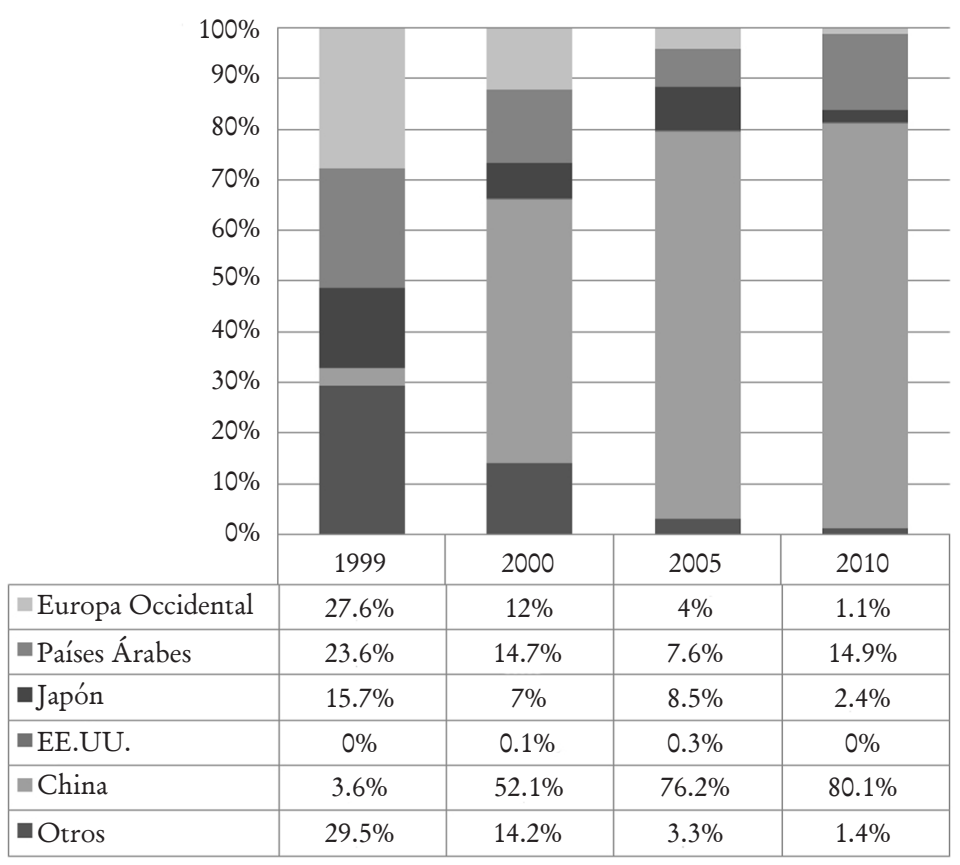

Fuente: World Bank, 2003, y sCBs, 2012.

Llama la atención que esta "lluvia de millones" no haya ido acompañada de un nuevo equilibrio del sector exterior, sino que hubiera déficits comerciales importantes. Esto no fue más que resultado del crecimiento sin precedentes de las importaciones como respuesta a la momentánea superación de la falta de liquidez. A finales de 2010, las importaciones se habían multiplicado por más de 10 (en dólares corrientes) respecto a la situación de 10 años antes. Hay pocos datos que avalen que el gasto militar haya tenido una influencia relevante en este fuerte crecimiento de las importaciones (gráfica 6). Por el contrario, éste fue interior en buena medida y tuvo que ver con el fortalecimiento del complejo industrial militar creado en la década anterior (HSBA, 2014). 
GRÁfICA 6. Evolución de la distribución de las importaciones por producto en 2000, 2004, 2008 y 2011

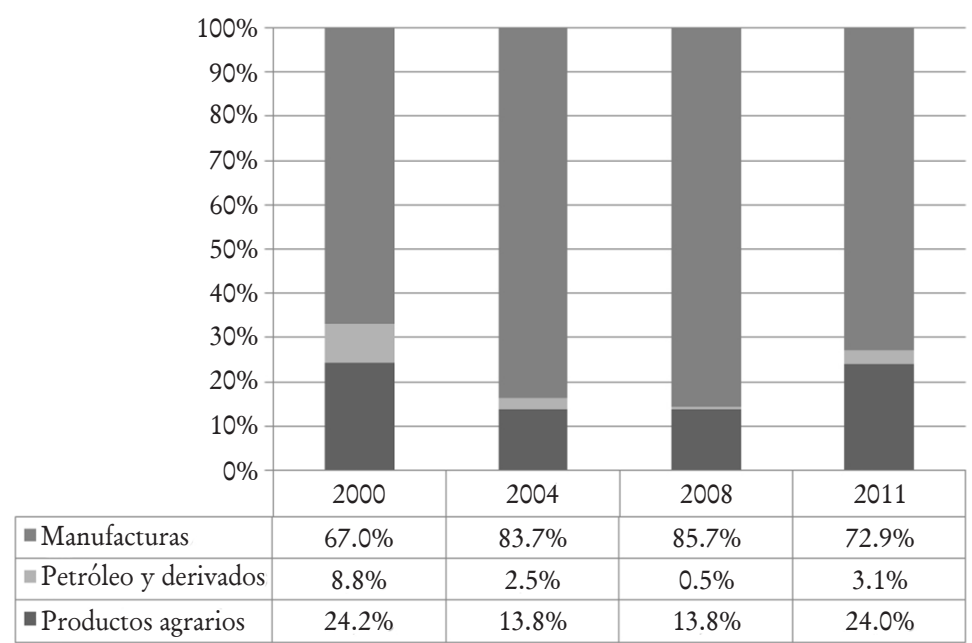
2009).

Fuente: elaboración propia a partir de datos del Banco Mundial (World Bank,

¿Por qué se disparó la necesidad de importar? Aunque el sector manufacturero no se estancó, sino que, por el contrario, creció a una tasa media anual acumulativa de $6 \%$ entre 2000 y 2010 - similar a la de crecimiento del PIB-, la demanda interior fue muy superior, y la importación de manufacturas llegó a representar más de $85 \%$ del total en 2008 . Con esto se observa cómo las dinámicas de crecimiento del PIB están asociadas a rápidos aumentos de importaciones en sectores como equipos de transporte, productos químicos, equipamiento eléctrico, electrodomésticos, equipos electrónicos, así como fibras textiles y plásticos, entre otros (World Bank, 2003, 2009 y 2012; sCBS, 2012). O, dicho de otra forma, el aumento de la renta interior provocó un cambio en el patrón de consumo, asociado a la compra de coches, electrodomésticos, etcétera, en buena medida importados. Además, se requería la inversión en ciertos procesos productivos, lo que significó también compra de maquinaria y equipos. 
Es el típico perfil de una economía dependiente: por un lado, está sujeta a una demanda externa poco diversificada; por otro, su aparato productivo no puede aprovechar por completo los aumentos de demanda, ya que los efectos multiplicadores potenciales se filtran rápidamente hacia el exterior (Prebisch, 1981; Furtado, 1989). Se desperdicia, por tanto, la "lluvia de millones" del petróleo para revertir la recurrente falta de liquidez, para generar capacidad interna de financiación o para reducir el endeudamiento exterior y, con ello, conseguir una posición de mayor fuerza en relación con las instituciones financieras internacionales.

Sin embargo, el enorme crecimiento de la renta implicó que el mercado sudanés se expandiera, lo que a su vez favoreció una diversificación de los proveedores internacionales. De esta forma, las importaciones pasaron a tener una estructura más variada. China era un importante abastecedor comercial, sobre todo de manufacturas (18\%), pero, en todo caso, se encontraba lejos de ser el proveedor dominante, como se observa en la gráfica 7.

Además, las importaciones crecieron en mayor medida porque (salvo en el caso de la industria bélica) no hubo ningún programa de sustitución de importaciones. Por ejemplo, en el sector agrario no se consiguió alcanzar el autoabastecimiento de producciones básicas. De esta manera, si $4.7 \%$ del total de las importaciones en 2004 correspondía a trigo y harina, en 2010 estos productos supusieron ya 11\% (Banco Mundial, 2015). Esto es especialmente significativo debido a las amplias posibilidades de desarrollo agrario del país y a las recurrentes situaciones de hambruna que se sufren en ciertas áreas (WFP, 2004; Prunier, 2005; Keen, 2008).

Es posible incluso que el aumento de los precios en el interior haya desincentivado la producción. Esto sería un primer indicador que apuntaría a problemas de "enfermedad holandesa", que pueden ser graves a mediano plazo en economías especializadas en la explotación de recursos naturales. $\mathrm{O}$, dicho en otros términos, Sudán comenzó a tener algunas características estructurales de las economías de corte extractivista (Gudynas, 2009; Svampa, 2011). Así, se generaron importantes rentas, pero se profundizó en las deficiencias estructurales que 
GrÁFICA 7. Evolución de la distribución de las importaciones en 2000, 2005 y 2010 según origen

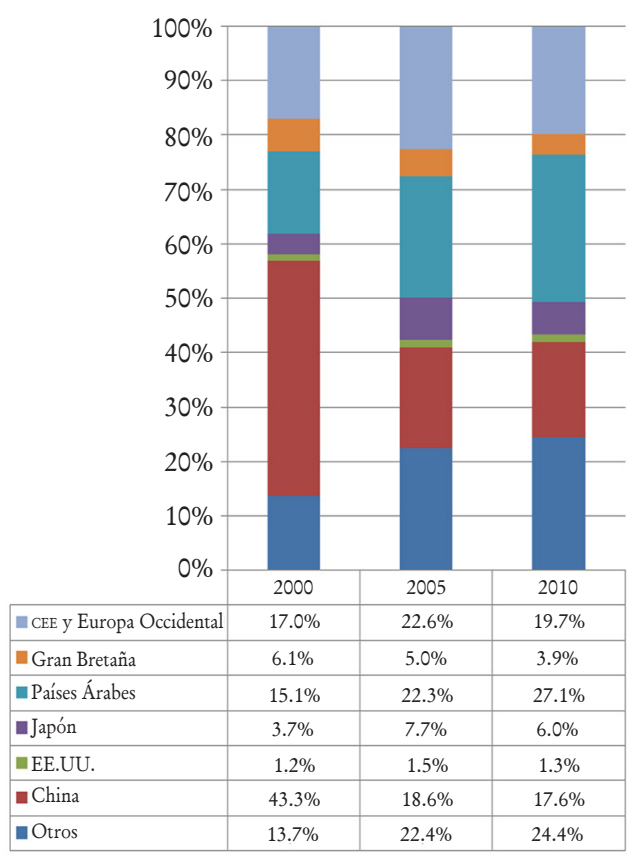

* CEE: Comunidad Económica Europea.

Fuente: SCBS, 2012.

no permitieron el acceso a bienes que deberían garantizarse a la población, como es el caso de una cesta básica de alimentos.

Así pues, los principios del tamakeen y la figh eldarura se aplican sólo parcialmente en lo referente a política comercial, y elementos que deberían entenderse como básicos en cualquier proceso de empoderamiento, como la soberanía alimentaria o el desarrollo de una base industrial propia, no desempeñaron un papel relevante en este periodo. China actuó como el primer financiador de la economía sudanesa y aportó un volumen creciente de manufacturas. Sin embargo, no desplazó del todo a los países europeos como fuente de aprovisionamiento industrial, ni se implicó en el desarrollo de sectores productivos propios 
más allá del petróleo ni en la construcción de infraestructuras (Bosshart, 2007).

\section{Las transformaciones en el papel del Estado}

El gobierno islamista estaba de acuerdo con algunas recetas neoliberales, en concreto con aquellas que propugnaban una pérdida del peso y la importancia del Estado en la economía (Al Turabi, 1983; Burr y Collins, 2003). En la gráfica 8 se observa cómo, durante la década de 1990, la formación bruta de capital fijo por parte del Estado no superó ningún año el 1\% del PIB. La actividad estatal, siguiendo una lógica muy cercana a los postulados del neoliberalismo, se basó en la creación de condiciones favorables para la afluencia de capitales privados, muchos de los cuales provenían de Asia en general, y de China en particular.

GRÁFICA 8. Participación en el PIB de la formación bruta de capital fijo del sector público

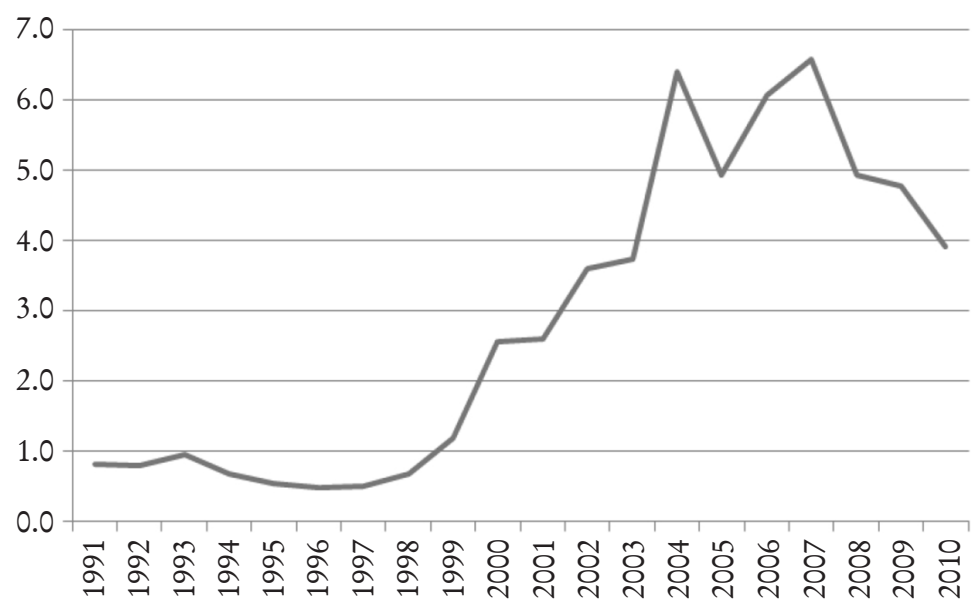
2012).

Fuente: elaboración propia a partir de datos del Banco Mundial (World Bank, 
El Estado también se retiró de la provisión de servicios públicos, como educación o sanidad. El gasto público en educación ascendió a $1.01 \%$ del PIB en 2000 , y el de sanidad, a $0.88 \%$, cifras que por sí solas hablan de la intensidad del proceso de privatización en estos dos sectores. Por ello, no hay un conflicto importante entre el gobierno sudanés y las instituciones financieras internacionales, en especial con el FMI. Ambas partes son partidarias de la aplicación de políticas de ajuste que reduzcan el papel del Estado a su mínima expresión. De hecho, la participación del gasto público en el PIB cayó de $26.7 \%$ en 1990 a $11.8 \%$ en 1999 . Esta reducción fue de la mano de una disminución de la presión fiscal, que se situó en $11.8 \%$ del PIB en 2000 (World Bank, 2003, 2009 y 2012). Con estos niveles de recaudación fiscal y en ausencia de ingresos procedentes del petróleo era prácticamente imposible para el Estado tener una mínima provisión de servicios públicos o ejercer un papel inversor directo.

De este modo, en 1995 más de 80\% del gasto en salud lo realizaban instituciones privadas (World Bank, 2003, 2009 y 2012). Los servicios los asumían, en su mayor parte, entidades islamistas (De Waal, 1997). Es decir, se produjo una particular forma de privatización de los servicios públicos que fortalecía la centralidad de las instituciones religiosas en general, e islamistas en particular, en la estructura social sudanesa. El concepto de caridad tuvo un papel cardinal en la justificación discursiva de esta transferencia de propiedad, al convertir la privatización en una vía especialmente efectiva de transformación microsocial (De Waal, 1997; Musa, 2001; Elnur, 2009). Sin embargo, no consiguió erradicar la pobreza ni la inseguridad alimentaria. Adicionalmente, este particular proceso de privatización fortaleció una definición excluyente de la identidad sudanesa. Aceptar la forma propuesta por el islamismo de entender la religión fue un factor muy relevante a la hora de garantizar el acceso a la educación y la sanidad.

Se asiste así a una redefinición del capitalismo sudanés a partir de la destrucción de los mecanismos de acumulación característicos del Estado desarrollista. Esto coincide con lo que algunos autores han denominado, en el contexto de economías desarrolladas, el rolling-back neoliberal (Peck y Tickell, 2002). 
Al mismo tiempo, se apuesta por un nuevo extractivismo como eje central de futuros procesos de crecimiento económico. El rápido impulso de la actividad petrolera afectó las cuentas públicas por dos vías: generó una dinámica de crecimiento del PIB que aumentó los ingresos públicos y, al encontrarse sujeta a distintos gravámenes específicos (siendo además públicas algunas empresas), obtuvo mejor recaudación. De esta forma, el petróleo fue la principal fuente de ingresos para el Estado.

En 2010, el petróleo generaba casi $65 \%$ del total de los ingresos públicos (OECD, 2012). Este mayor acceso al financiamiento le permitió al Estado llevar a cabo de mejor manera algunas funciones, y se observa cómo emergió un nuevo modelo de intervención pública sobre la base de la hibridación de elementos islamistas, neoliberales y extractivistas (World Bank, 2003, 2009 y 2012).

\section{Las lógicas de conflicto armado y el papel del petróleo}

Las tensiones entre el norte y el sur en Sudán son seculares y se remontan, cuando menos, a la independencia del país en 1956. Hay grandes diferencias en la forma de construir la identidad entre el norte, profundamente musulmán y vinculado al mundo árabe, y el sur, con mayor influencia del colonialismo inglés y mayoritariamente cristiano-animista (Beswick, 2004).

El petróleo siempre ha condicionado la guerra civil entre norte y sur, aunque de modo muy diferente. Desde la década de 1970 se sabe de importantes reservas que, de facto, actuaron como garantía de los diversos préstamos otorgados al gobierno sudanés por la banca y las instituciones financieras internacionales. No obstante, el papel del petróleo en la financiación del Estado sudanés en general y del esfuerzo bélico que para éste suponía la guerra civil en el sur del país fue cada vez mayor a partir de 1990.

Desde ese momento empezaron a otorgarse derechos de explotación de distintas zonas. En el mapa 3 se observan las principales concesiones petrolíferas de Sudán en 2007, que se dividían en 15 bloques. Del 1 al 5a constituyen las primeras 
MAPA 3. Concesiones petrolíferas en 2007

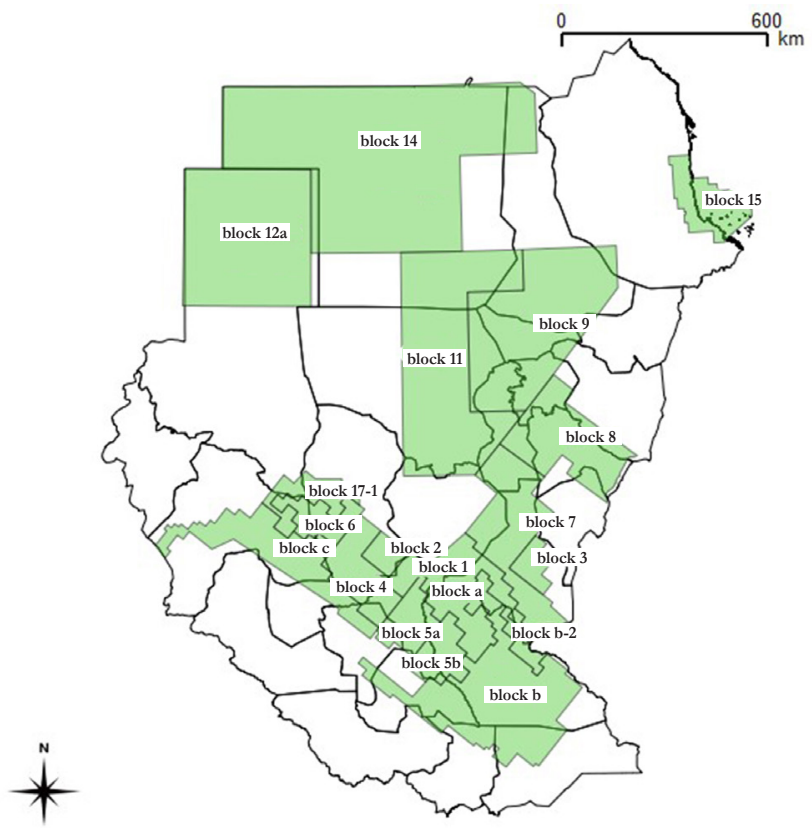

Fuente: EIA, 2007, y elaboración propia.

zonas exploradas, debido a que en ellas se encontraban las mayores reservas. De hecho, su puesta en marcha abarcó la década de 1990. Evidentemente, en ello influyeron también los avances en las técnicas de prospección, capaces de detectar las reservas ocultas, aun cuando hay evidencias de que se sabía de su existencia al menos desde finales de los años setenta (Human Rights Watch, 2003).

Los bloques del 1 al 5a no sólo se encuentran en la frontera entre norte y sur del país, sino que, como lo muestra el mapa 4, el área se caracteriza por la coexistencia de una multitud de etnias sureñas, en particular las de los dinka, los nuer y los luo. Todas con identidades específicas enfrentadas a la árabemusulmana dominante y que han mantenido relaciones histó- 
ricamente conflictivas con el Estado (Jok y Hutchinson, 1999). Por esta razón, la puesta en explotación de estos yacimientos implicó necesariamente el aumento del control del gobierno central sobre estas áreas, lo que, dada la situación de guerra civil, implicaba mayor gasto y esfuerzo militar (Patey, 2007). Además, debido a que el Estado sudanés y los grupos sociales (del norte) que lo controlan se han apropiado de la mayor parte de las rentas derivadas del petróleo, esta apropiación se ha convertido en fuente adicional de conflicto (Reeves, 2002). La construcción de infraestructuras asociadas al petróleo ha propiciado la aparición de crecientes problemas socioambientales y, con ello, la intensificación de la lucha armada (Jok y Hutchinson, 1999).

Estos momentos críticos han sido utilizados por las guerrillas del sur para ampliar su base social. Frente a ello, siguiendo los procesos generales de liberalización económica dominantes en el país, ha habido una creciente privatización de la guerra, así como un uso de fuerzas paramilitares y formas de represión no convencionales contra los movimientos rebeldes (Human Rights Watch, 2003). La posición de China en este punto es contradictoria. Por un lado, basa su política exterior en general, y africana en particular, en el principio de "no intervención en asuntos internos". No obstante, su aplicación estricta implicaría dejar de explotar los recursos petrolíferos, lo que iría en contra de sus intereses. Por ello, en última instancia e indirectamente, el flujo monetario derivado del petróleo y la importancia de China en su explotación y posterior consumo han impulsado la violencia al "liberar" los yacimientos de elementos rebeldes para extraer el crudo. Además, ha apoyado activamente con asistencia tecnológica y cesión de patentes el desarrollo del Complejo Industrial Militar. Todo ha favorecido al nuevo Estado islamista de corte neoliberal a partir de los años noventa.

Un ejemplo de la violencia estructural es la sucesión de hambrunas, entre las que caben citarse tanto las de 1990-1991 y 1998 en Bahr el Ghazal, que afectaron mayoritariamente a población dinka (De Waal, 1997; Keen, 2008), como la de Darfur en 1990 y a partir de 2003, o las de 2009 y 2010 en Ecuatoria Oriental, Nilo Azul o Jonglei (Prunier, 2005; Daly, 2007). Estas 


\section{MAPA 4. Áreas de las primeras concesiones petrolíferas y sus divisiones étnicas en 1998}

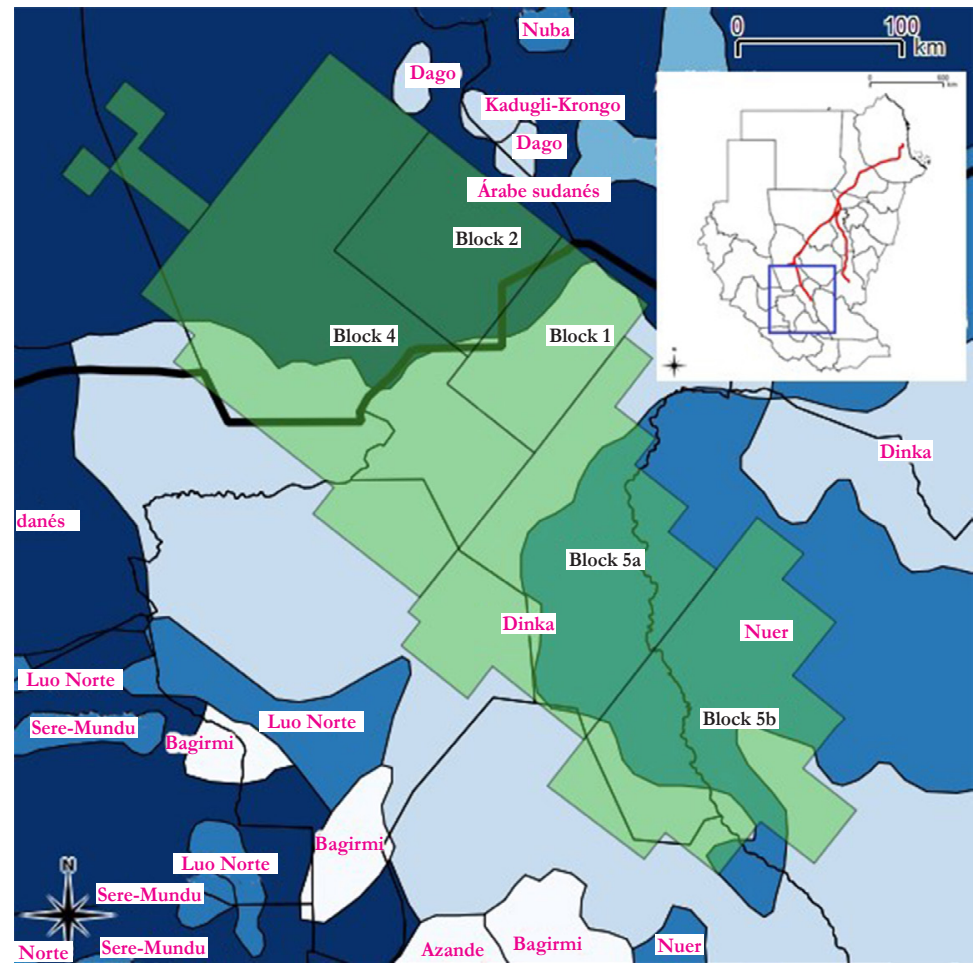

Fuente: EIA, 2007, y elaboración propia.

hambrunas no tuvieron su origen en catástrofes naturales y resultan totalmente incomprensibles en un contexto de boom petrolero que ha multiplicado por más de 10 la entrada de divisas en Sudán en una década. Deben entenderse más bien como continuación de una estrategia de represión que, en muchos casos, ha estado marcada por la localización de los yacimientos de crudo. Así pues, la renta petrolera no sólo no ha evitado la inseguridad alimentaria, sino que, como se ve en el mapa 5 , en amplias áreas del país ésta se ha vuelto crónica. De esta manera, la generación de hambrunas se ha convertido en una manera 
MAPA 5. Proporción de hogares con inseguridad alimentaria severa en octubre/noviembre de 2010

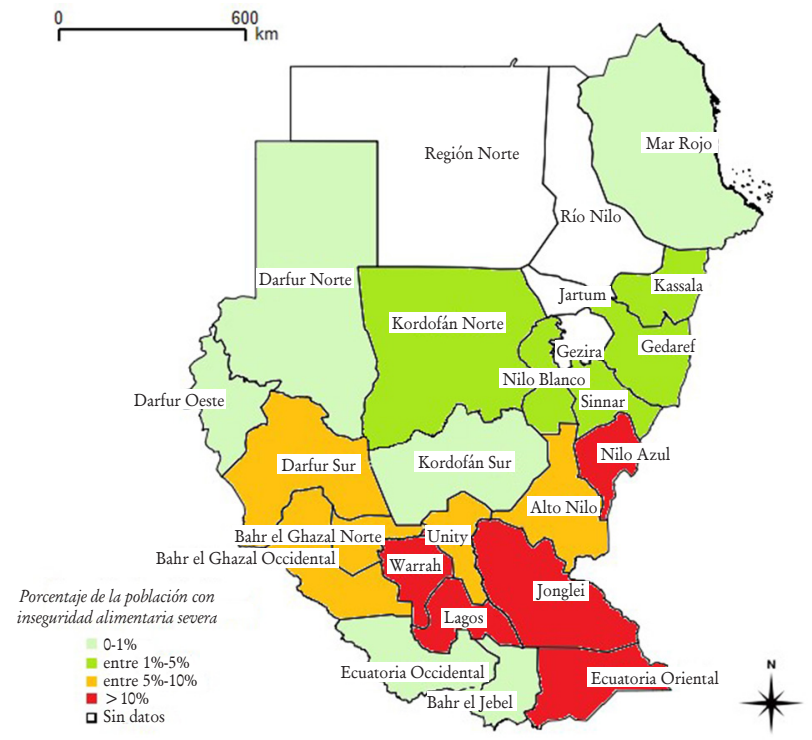

Fuente: WFP-FAO, 2011, y elaboración propia.

de hacer la guerra por medios no convencionales, acorde con el creciente uso de grupos paramilitares.

\section{Conclusiones}

La evolución de Sudán en los últimos 25 años no puede ser entendida sin cuatro procesos concurrentes: los efectos del neoliberalismo en África y el mundo árabe, el proyecto político islamista, la extracción masiva de petróleo y los cambios en el marco geoestratégico global marcado por el creciente papel de China. Como se ha analizado, el gobierno islamista promovió en los años noventa un roll-back del Estado, similar al observable en los años ochenta en muchas economías desarrolladas (Peck y Tickell, 2002). La privatización masiva de la educación 
y la sanidad fue fundamental para que los grupos vinculados al islamismo ganasen centralidad en la estructura social sudanesa.

Por la relevancia de la privatización, la reducción impositiva y la globalización, es evidente que la experiencia sudanesa sigue las medidas fundamentales del Consenso de Washington, entendido como el modelo de gestión económica defendido por el FMI, el Banco Mundial y el Departamento del Tesoro de Estados Unidos (Williamson, 1993). En este sentido, paradójicamente, islamismo y neoliberalismo van a coincidir en la promoción de muchas políticas, aunque el propósito último es, sin duda, muy diferente.

El islamismo precisaba también de crecimiento económico y rentas monetarias para su consolidación. Éste fue esencialmente el papel del petróleo, que consiguió movilizar inversiones y generar rentas monetarias fundamentales para el funcionamiento de la economía del país. China ha sido muy importante en este proceso. En los años noventa, tuvo un gran protagonismo como inversor, no sólo en la puesta en explotación de los pozos, sino también en un conjunto de inversiones asociadas, como oleoductos y refinerías. En la década de 2000, China intensificó su carácter de inversor y se convirtió en casi el único destino internacional del petróleo extraído. No obstante, tanto la aproximación del gobierno islamista a China como la política del gigante asiático respecto a Sudán han estado marcadas por el pragmatismo.

En los últimos años, China ha enfocado crecientemente su política exterior en criterios normativos, concretados en el Consenso de Beijing, cuyo efecto ha sido especialmente importante en África (Galchu, 2018). Uno de los tres principios que lo sustentan es el respeto y el reconocimiento de la soberanía de los distintos países a la hora de buscar su propio modelo de desarrollo y de orden social (Ramo, 2004). En este sentido, la máxima de "no injerencia en asuntos internos" y el tamakeen islamista han demostrado dialogar relativamente bien durante un periodo que ya supera los 20 años. China, además, ha actuado como contrapeso de Estados Unidos al permitir una ampliación del marco de lo políticamente posible.

Adicionalmente, la "flexibilidad" de la política china le ha permitido a ese país adaptarse al cambiante y a veces caótico 
escenario sudanés. Sin embargo, otros principios del Consenso de Beijing, como la apuesta por cambios cualitativos en la gestión socioeconómica que vayan asociados a una mayor sostenibilidad o equidad social, difícilmente ocurren en el caso sudanés. En realidad, la superación de los problemas estructurales de la economía sudanesa (extraversión, desarticulación y dualidad) no ha sido considerada como un objetivo por los sucesivos gobiernos.

La mejor prueba del carácter poco "armonioso" del modelo sudanés es la sucesión de varias hambrunas, ya que, en un país con fuerte potencial agrario, no se ha avanzado significativamente en la mejora del autoabastecimiento de alimentos básicos. En los años posteriores, la mayor inseguridad alimentaria y el mayor riesgo de hambruna en el norte han seguido concentrándose en el Gran Darfur, Kordofán Sur y Nilo Azul, mientras que en el sur el hambre ha afectado con mayor dureza a Jonglei, Warrab, Lagos y Ecuatoria Oriental. Un indicador de que no se corrigió la desarticulación que afectaba la estructura productiva sudanesa es que, durante la década de 2000, hubo escasez generalizada de alimentos en ciertas zonas del país, al tiempo que se disparaban los ingresos del petróleo.

Tampoco se observan progresos relevantes en otro de los principios del Consenso de Beijing: el desarrollo de una economía basada en la innovación. La única novedad en la economía sudanesa en el periodo analizado es la extracción masiva de petróleo que supuso que el PIB aumentara en 100.9\% en términos reales de 1999 a 2011 y el PIB per cápita en 61.1\% (World Bank, 2003, 2009 y 2012). Si semejante crecimiento de las rentas no ha sido capaz de detener la progresión de las hambrunas y ha implicado una profundización de las lógicas de conflicto armado, ¿puede ser calificado este proceso como "empobrecedor"? ¿Puede considerarse Sudán un caso extremo de dinámicas más amplias de acumulación por desposesión? (Harvey, 2004).

En todo caso, se observa una redefinición de la física del poder y del desarrollo (Ramo, 2004). En este contexto, Estados Unidos ha quedado totalmente desplazado ante el emergente vínculo entre China y el islamismo sudanés. Los capitales norteamericanos no han actuado ni como inversores ni como 
distribuidores ni como consumidores del petróleo extraído. En realidad, la política exterior norteamericana, debido a las fuertes tensiones con el gobierno islamista concretadas en sanciones económicas, ha contribuido al acercamiento chino-sudanés. Por eso no sorprende el creciente alineamiento de Estados Unidos con los grupos rebeldes del sur, ni su apoyo a la partición del país en 2011. Muchos elementos explican esta división; el principal es el enfrentamiento civil crónico, que se remonta a la independencia del país en 1956. Sin embargo, la posición adoptada por Estados Unidos en su lucha con China por la hegemonía en África es, sin duda, un factor muy importante (Taylor 2006; Mohan y Power, 2009).

En definitiva, en Sudán, durante el periodo 1989-2011, se han ensayado, como en un laboratorio, las formas y los efectos de la hibridación entre neoliberalismo, islamismo y extractivismo bajo la égida de un todavía hegemónico Consenso de Washington frente a un emergente y aún incierto Consenso de Beijing.

Dirección institucional de los autores:

Alice Salomon Hocbschule Berlin

Alice-Salomon-Platz 5

12627, Berlin, Germany

Facultad de Ciencias Económicas y Empresariales

Universidad de Sevilla

Avda. Ramón y Cajal, s/n

41018, Sevilla, España

\section{Referencias}

Al Turabi, H. A. (1983). The Islamic State. En J. L. Esposito (Ed.), Voices of resurgent Islam (pp. 241-251). Nueva York: Oxford University Press.

Banco Mundial. (2015). Banco de datos mundial. Indicadores del desarrollo mundial. Recuperado de https://databank.bancomundial. org/data/source/world-development-indicators 
BAYAT, A. (2006). Islamism and social movement theory. Third World Quarterly, 26(6), 891-908. https://dx.doi.org/10.1080/ 01436590500089240

BerHANu, D. G. (2011). Institutions and investment in Sudan. Socioeconomic and institutional foundations of reconstruction and development. Münster: LIT.

BEswick, S. (2004). Sudan's blood memory. The legacy of war, ethnicity, and slavery in South Sudan. Rochester, NY: University of Rochester Press.

BHASKAR, R. (2011). Reclaiming reality. A critical introduction to contemporary philosophy. Londres: Routledge.

Bosshart, P. (14 de mayo de 2007). China's role in financing African infrastructure. Berkeley, CA: International Rivers Network. Recuperado de https://www.internationalrivers.org/sites/default/ files/attached-files/chinaeximbankafrica.pdf

Brenner, N., Peck, J. y Theodore, N. (2010). Variegated neoliberalization: Geographies, modalities, pathways. Global Networks, 10(2), 182-222. https://dx.doi.org/10.1111/j.1471-0374.2009. 00277.x

Burr, J. M. y Collins, R. O. (2003). Revolutionary Sudan. Hasan alTurabi and the Islamist State, 1989-2000. Leiden: Brill.

DALY, M. W. (2007). Darfur's sorrow. A history of destruction and genocide. Cambridge: Cambridge University Press.

De WaAl, A. (1997). Famine crimes. Politics and the disaster relief industry in Africa. Londres: African Rights and the International African Institute.

EL-AFFENDI, A. (1991). Turabi's revolution. Islam and power in Sudan. Londres: Grey Seal Books.

ELnuR, I. (2009). Contested Sudan. The political economy of war and reconstruction. Londres: Routledge.

FurTado, C. (1989). La fantasía organizada. Bogotá: Tercer Mundo.

Galchu, J. (2018). The Beijing consensus versus the Washington consensus: The dilemma of Chinese engagement in Africa. African Journal of Political Science and International Relations, 12(1), 1-9. https://dx.doi.org/10.5897/AJPSIR2016.0920

Gual, M. A. y Norgaard, R. B. (2010). Bridging ecological and social systems coevolution: A review and proposal. Ecological Economics, 69(4), 707-717. https://doi.org/10.1016/j.ecolecon.2008. 07.020

GudynAs, E. (2009). Diez tesis urgentes sobre el nuevo extractivismo. Contextos y demandas bajo el progresismo sudamericano actual. En J. Schuldt et al., Extractivismo, política y sociedad (pp. 187-225). 
Quito: El Centro Andino de Acción Popular-Centro Latinoamericano de Ecología Social. Recuperado de http://extractivismo. com/wp-content/uploads/2016/06/GudynasExtractivismoSociedadDesarrollo09.pdf

Harvey, D. (2004). El nuevo imperialismo. Madrid: Akal.

Harvey, D. (2007). Breve historia del neoliberalismo. Madrid: Akal. Human Rights Watch (2003). Sudan, oil, and buman rights. Nueva York, NY: Human Rights Watch. Recuperado de https://www. hrw.org/reports/2003/sudan1103/sudanprint.pdf

Human Security Baseline Assessment (HSBA). (2014). The Military Industry Corporation (MIC), Ginebra: Small Arms Survey. http:// www.smallarmssurveysudan.org/fileadmin/docs/facts-figures/ sudan/HSBA-MIC-Open-Source-Review-2014.pdf

Jок, M. J. (2001). War and slavery in Sudan. Filadelfia, PA: University of Pennsylvania Press.

JoK, M. J. y Hutchinson, S. E. (1999). Sudan's prolonged second civil war and the militarization of Nuer and Dinka ethnic identities. African Studies Review, 42(2), 125-145. https://dx.doi. org/10.2307/525368

KeEN, D. (2008). The benefits of famine: A political economy of famine and relief in soutbwestern Sudan 1983-1989. Oxford: James Currey.

Kobryn, J. (2004). Oil and politics: Talisman energy and Sudan. New York University Journal of International Law and Politics, 36(2), 425-455. Recuperado de https://faculty.wharton.upenn.edu/ wp-content/uploads/2012/05/nyujilp.pdf

LARGE, D. (2007). China and the changing context of development in Sudan. Development, 50(3), 57-62. https://dx.doi.org/10.1057/ palgrave.development.1100405

LARGE, D. (2008). China and the contradictions of 'non-interference' in Sudan. Review of African Political Economy, 35(115), 93-106. https://dx.doi.org/10.1080/03056240802011568

Larner, W. (2003). Neoliberalism? Environment and Planning D: Society and Space, 21(5), 509-512. https://dx.doi.org/10.1068/ $\mathrm{d} 2105 \mathrm{ed}$

LAWsON, T. (1997). Economics and reality. Londres: Routledge. Lawson, T. (2003). Reorienting economics. Londres: Routledge.

LE BILlon, P. (2001). The political ecology of war: Natural resources and armed conflicts. Political Geography, 20(5), 561-584. https:// doi.org/10.1016/S0962-6298(01)00015-4

Middle East Economic Survey. (2003). First oil from CNPC's block 6 to lift Sudan production in 2004. Sudan Tribune. Recuperado 
de http://www.sudantribune.com/spip.php?iframe\&page $=$ imprimable\&id article $=1012$

Mohan, G. y Power, M. (2009). Africa, China and the 'new' economic geography of development. Singapore Journal of Tropical Geography, 30(1), 24-28. https://dx.doi.org/10.1111/j.14679493.2008.00352.x

Musa, E. A. (2001). Sudan Structural Adjustment Programme (SSAP): Some implications for labour in the formal sector. Cabiers africains d'administration publique, (56), 67-76. Recuperado de http:// unpan1.un.org/intradoc/groups/pblic/documents/CAFRAD/ UNPAN017581.pdf

Nelson, R. (2006). Evolutionary social science and universal Darwinism. Journal of Evolutionary Economics, 16(5), 491-510. https:// dx.doi.org/10.1007/s00191-006-0025-5

Organización para la Cooperación y el Desarrollo Económicos (OECD). (2012). Sudan. En African Economic Outlook 2012: Promoting Youth Employment. París: OECD Publishing. https://doi. org/10.1787/aeo-2012-55-en

Parello-Plesner, J. y Duchâtel, M. (2014). China in deep in the oil-rich Sudans. Adelphi Series, 54(451), 125-144. https://dx.doi. org/10.1080/19445571.2014.1047147

Patey, L. A. (2007). State rules: Oil companies and armed conflict in Sudan. Third World Quarterly, 28(5), 997-1016. https://dx.doi. org/10.1080/01436590701371728

Peck, J. y Tickell, A. (2002). Neoliberalizing space. Antipode, 34(3), 380-404. https://dx.doi.org/10.1111/1467-8330.00247

Prebisch, R. (1981). Capitalismo periférico: crisis y transformación. México: Fondo de Cultura Económica.

Prunier, G. (2005). Darfur. The a mbiguous genocide. Londres: Hurst and Company Publishers.

Ramo, J. C. (2004). The Beijing consensus. Londres: Foreign Policy Centre.

Reeves, E. (2002). Oil development in Sudan. Review of African Political Economy, 29(91), 167-169. https://dx.doi.org/10.1080/ 03056240208704600

Rone, J. (2003). Sudan: Oil \& war. Review of African Political Economy, 30(97), 504-510, 478.

Sayer, A. (1995). Radical political economy: A critique. Oxford: Blackwell.

Sharfi, M. H. (2015). Sudan's radical foreign policy agenda in the 1990s: An overview of implications. Contemporary Arab Affairs, 8(4), 523-534. https://dx.doi.org/10.1080/17550912.2015.107 7611 
Sudan Central Bureau of Statistics (SCBS). (2012). Sudan Foreign Trade Statistics Summary 2000-2010. Jartum: Sudan Central Bureau of Statistics.

Svampa, M. (2011). Extractivismo neodesarrollista y movimientos sociales: ¿un giro ecoterritorial hacia nuevas alternativas? En Más allá del desarrollo (pp. 185-216). México: Fundación Rosa Luxembourg.

TAYLOR, I. (2006). China's oil diplomacy in Africa. International Affairs, 82(5), 937-959. https://dx.doi.org/10.1111/j.1468-2346.2006. 00579.x

U.S. Energy Information Admistration (EIA). (2007). Country Analysis Briefs. Sudan and South Sudan. Recuperado de http://www. ecosonline.org/others/2007/EIA\%20Country\%20Report. pdf

U.S. Energy Information Admistration (EIA). (2013). Country Analysis Briefs. Sudan and South Sudan. Recuperado de https://www. eia.gov/beta/international/analysis includes/countries_long/ Sudan_and_South_Sudan/sudan.pd $\bar{f}$

WARBURG, G. (2003). Islam, sectarianism and politics in Sudan since the Mabdiyya. Londres: Hurst \& Co.

Williamson, J. (1993). Democracy and the "Washington consensus". World Development, 21(8), 1329-1336. https://dx.doi. org/10.1016/0305-750X(93)90046-C

Woodward, P. (1994). Sudan: A new political character? En C. Gurdon (Ed.), The horn of Africa (pp. 76-89). Nueva York, NY: St. Martin Press.

World Bank. (junio de 2003). Sudan. Stabilization and reconstruction. Country economic memorandum. Vol. 1: Main text (Report No. 24620-SU). Washington, D.C.: World Bank. Recuperado de http://documents.vsemirnyjbank.org/curated/ $\mathrm{ru} / 440091468777571140 / \mathrm{pdf} / \mathrm{multiO}$ page.pdf

World Bank. (diciembre de 2009). Sudan. The road toward sustainable and broad-based growth. Washington, D.C.: World Bank. Recuperado de http://documents.worldbank.org/curated/ en/337451468130798599/pdf/547180ESWOP07610publicOdist ribution.pdf

World Bank. (diciembre de 2012). Sudan Economic Brief: Recent Economic Developments. 2nd Semester 2012. Washington, D.C.: World Bank. Recuperado de http://documents.worldbank.org/ curated/en/809251468102891015/pdf/754930BRIOSuda00Box3 74332B00PUBLIC0.pdf

World Food Programme (wfr). (2004). Sudan Annual Needs Assess- 
ment 2003/2004. Food security report: Regional outlines E recommendations. Jartum: World Food Programme.

World Food Programme. (wFP)-Food and Agriculture Organization (FAO). (2011). Government of Sudan and FAO/WFP crop and food security assessment mission to the 15 Northern States of Sudan. Recuperado de https://reliefweb.int/sites/reliefweb.int/files/resou rces/86D7B8B52E7C39B9C125783100358BE8-Full_Report.pdf 
